\title{
Loss of Chromosome $Y$ and Its Potential Applications as Biomarker in Health and Forensic Sciences
}

\author{
Bela Barros ${ }^{a, b} \quad$ Mariana Morais $^{a}$ b $\quad$ Ana Luísa Teixeira ${ }^{a} \quad$ Rui Medeiros ${ }^{a-e}$ \\ ${ }^{a}$ Molecular Oncology and Viral Pathology Group, Portuguese Oncology Institute of Porto Research Center (CI-IPOP),

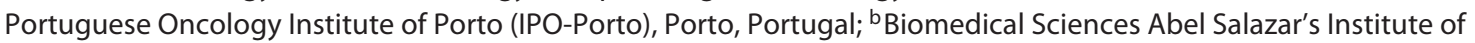 \\ University of Porto (ICBAS), Porto, Portugal; ' ${ }^{C}$ Faculty of Medicine, University of Porto, Porto, Portugal; \\ ${ }^{\mathrm{d}}$ Research Department, Portuguese League Against Cancer, Porto, Portugal; ${ }^{\circ}$ CEBIMED, Faculty of Health Sciences, \\ Fernando Pessoa University, Porto, Portugal
}

\section{Keywords}

Biomarker · Forensic and health applications · Loss of

chromosome $Y \cdot$ Mosaicism · Y chromosome

\begin{abstract}
Loss of chromosome $Y$ (LOY) is a mosaic aneuploidy that can be detected mainly in blood samples of male individuals. Usually, LOY occurrence increases with chronological age in healthy men. Moreover, recently LOY has been reported in association with several diseases, such as cancer, where its frequency is even higher. The $Y$ chromosome is one of the shortest chromosomes of the human karyotype, and it is crucial for correct male development. This chromosome has functions beyond the male reproductive system, and loss of its genes or even LOY can have consequences for the male body that are yet to be elucidated. Analyses of the Y chromosome are largely applied in forensic contexts such as paternity testing, ancestry studies, and sexual assault cases, among others. Thus, LOY can be a disadvantage, limiting laboratory methods and result interpretation. However, as an advantage, LOY detection could be used as a biological age biomarker due to its association with the aging process. The potential application of LOY as biomarker highlights the
\end{abstract}

karger@karger.com

(c) 2020 S. Karger AG, Basel

www.karger.com/cgr

Karger! necessity to clarify the molecular mechanism behind its occurrence and its possible applications in both health and forensic studies.

(c) 2020 S. Karger AG, Basel

Loss of chromosome Y (LOY) is a hypodiploidy ( $2 n-$ 1 ), and it is mainly described as a mosaic loss in blood cells of male individuals. Hence, other events, such as microdeletions, are not considered as LOY [Dumanski et al., 2017]. LOY is the most common non-physiological postzygotic genetic alteration in human beings [Forsberg, 2017; Forsberg et al., 2017]. In fact, LOY is a normal aging process since it is present in healthy individuals and its percentage rises in the course of time [Guttenbach et al., 1995]. Moreover, Forsberg et al. [2014] observed a mean decrease of 5.5 years in the lifespan of men who presented LOY in their blood cells. Studies have also demonstrated an association between LOY and the development of diseases such as Alzheimer disease, autoimmune diseases (autoimmune thyroiditis and binary cirrhosis), schizophrenia, cardiovascular events, and a wide number of cancers, i.e., prostate cancer, acute myelogenous leukaemia, oesophageal carcinoma, gastric cancer, bladder cancer, renal cell carcinoma, and testicular germ cell tumour 
(TGCT) [Holmes et al., 1985; Castedo et al., 1992; Hunter et al., 1993; Guttenbach et al., 1995; Sauter et al., 1995; Brunelli et al., 2003; Gilgenkrantz, 2008; Klatte et al., 2009; Persani et al., 2012; Lleo et al., 2013; Dumanski et al., 2016; Noveski et al., 2016; Forsberg, 2017; Forsberg et al., 2017; Haitjema et al., 2017; Hirata et al., 2018]. Therefore, LOY can be seen as a biological age marker, and its presence has potential to be used as a predictor biomarker of male age-related diseases [Dumanski et al., 2016]. On the other hand, the analysis of the Y chromosome is commonly performed in forensic studies, and the application of LOY analysis can provide both disadvantages and advantages to this field. On the one hand, LOY may interfere with forensic analysis of male samples because the detection of the Y chromosome could be compromised. Simultaneously, LOY can be of use in forensic cases by adding information which can be brought to the law court as well.

\section{Structure of the Y Chromosome}

The Y chromosome was first reported in 1905, when Nettie Stevens deduced that sex was determined by the presence or absence of a chromosome that she named "Y" [Gilgenkrantz, 2008]. But only in 1990, Andrew Sinclair and his team discovered the sex-determining region $\mathrm{Y}$ $(S R Y)$ in this chromosome - a gene that triggers the male phenotype during embryonic development [Sinclair et al., 1990].

In 1985, two additional regions of the Y chromosome were identified: the pseudoautosomal regions (PAR). These regions are present on both the $\mathrm{X}$ and the $\mathrm{Y}$ chro-

Fig. 1. A full map of the Y chromosome with its 47 MSY proteincoding genes, their location and tissue expression. X-homologous genes are in blue, Y-specific genes are in black. $S R Y$ and TSPY are in blue because of the existence of some similarity to SOX3 and TSPX sequences on the X chromosome [Skaletsky et al., 2003; Lau et al., 2019]. Underlined in different colours are 3 different classes of genes: green, X-degenerate genes; red, ampliconic genes; and purple, X-transposed genes. Over the years, MSY genes were added to the Y chromosome map, so the genes are numbered by superscripts 1 to 8 , corresponding to the years of the articles when they were first added to the map: 1994, 1995, 1997, 2000, 2001, 2003, 2012, and 2015 respectively. The TSPY family genes include TSPY1, 2, 3, 4, 8, and 10 [Kido and Lau, 2015]. The RBM family comprises RBMY1B, A1,D,E, F, and $J$ genes. PRKY, GYG2P1, $C Y$ orf $15 A / B$ (also known as TXLNGY), and PRYP3/4 are not included in the map because they are pseudogenes [Kido and Lau, 2015; Maan et al., 2017]. USP9Y, DDX3Y, TMBS4Y, KDM5D, mosome but behave like autosomes and recombine during meiosis [Mangs and Morris, 2007]. PAR1 is located at the ends of the short arms (Xp and Yp) and PAR2 at the ends of the long arms (Xq and Yq) [Cooke et al., 1985; Freije et al., 1992]. Since the Y PARs have the highest homology with the PARs of the $\mathrm{X}$, they are the only regions of the $\mathrm{Y}$ chromosome undergoing crossing over in meiosis [Jobling and Tyler-Smith, 2000; Kumar and Sharma, 2017]. A third PAR (PAR3), approximately $2.3 \mathrm{Mb}$ in length, was identified in 2013; it shares more than $98 \%$ sequence homology with Xq21.3 [Veerappa et al., 2013]. However, its functional significance is yet unknown, since the Y chromosomal PAR3 was only identified in $2 \%$ of the general population [Colaco and Modi, 2018].

The PARs represent only 5\% of the Y chromosome. The remaining portion (95\%) does not recombine and is called "non-recombinant region Y" (NRY) or "male-specific region of the Y chromosome" (MSY) [Lahn and Page, 1997]. Until now, 47 MSY genes have been reported (Fig. 1). Previous attempts of MSY gene classification were based on the degree of homology with the X chromosome or the pattern of expression (testis-specific or ubiquitous) [Lahn and Page, 1997; Jobling and TylerSmith, 2000]. Nowadays, the most accepted classification divides MSY genes into 3 classes: X-transposed, X-degenerate, and ampliconic (Fig. 1) [Skaletsky et al., 2003]. The $\mathrm{X}$-transposed genes share $99 \%$ identity with the X chromosome (Xq21) [Skaletsky et al., 2003]. The X-degenerate elements are composed of pseudogenes or single-copy genes with $60-96 \%$ homology to X-linked genes [Skaletsky et al., 2003; Ginalski et al., 2004; Jangravi et al., 2013; Kumar and Sharma, 2017]. The ampliconic class genes are similar in length to the other MSY genes and

PRORY, and DAZ1 are also known as DFFRY, DBY, TB4Y, SMCY, CYorf17, and SPGY, respectively [Johansson, 2015; Kido and Lau, 2015; Maan et al., 2017]. The heterochromatin regions, represented by white cuts in the schematic chromosome image, include the centromere, the proximal ( $\sim 400 \mathrm{~kb}$ interrupting the euchromatic region on Yq11.22) and the distal Yq arm [Skaletsky et al., 2003]. Genes and expression patterns from PAR1 and PAR2 regions are not shown in this figure, and the recently discovered PAR3 is represented by the X-transposed genes on Yp11.2 (TGIF2LY and PCDH11Y) [Veerappa et al., 2013]. a Includes testis, prostate, kidney (cortex), adrenal gland, blood, spleen, liver, stomach, transverse colon, terminal ileum, pancreas, oesophagus, coronary artery, heart (left ventricle and atrial appendage), lung, thyroid, brain (cerebellum and cortex), skeleton muscle and adipose tissues. b Also expressed in other tissue(s), but testis expression has higher levels.

(For figure see next page.) 


\begin{tabular}{|c|c|c|c|c|}
\hline Arm & $\begin{array}{l}\text { Loci (Jangravi } \\
\text { et al. 2013) }\end{array}$ & $\begin{array}{l}\text { Protein coding-Gene } \\
\text { (Lahn and Page 1997; Jangravi et al. } \\
\text { 2013; Kido and Lau 2015; Maan et } \\
\text { al. 2017; Tilford et al. 2001) }\end{array}$ & Tissues where there is expression of the gene & Ref. \\
\hline \multirow[t]{10}{*}{ Yp } & Yp 11.32 & PAR1 & & $\begin{array}{l}\text { (Jangravi et al. 2013; } \\
\text { Skaletsky et al. 2003) }\end{array}$ \\
\hline & Yp 11.31 & SRY ${ }^{1 *}$ & Testis, Adrenal Gland, Oesophagus and Adipose & (Maan et al. 2017) \\
\hline & & RPS4Y1 & Ubiquitously expressed ${ }^{a}$ & (Maan et al. 2017) \\
\hline & & $Z F Y^{1}$ & Ubiquitously expressed ${ }^{a}$ & (Maan et al. 2017) \\
\hline & Yp 11.2 & TGIF2LY & Testis & (Maan et al. 2017) \\
\hline & & $\overline{\mathrm{PCDH11Y^{4 }}}$ & $\begin{array}{l}{ }^{a} \text { except Blood, Liver, Pancreas, Heart (Left ventricle) and skeletal } \\
\text { muscle }\end{array}$ & (Maan et al. 2017) \\
\hline & & TSPY $^{8}$ & Testis & (Maan et al. 2017) \\
\hline & & $\overline{A M E L Y^{1}}$ & Testis, Pancreas, Thyroid and Teeth & (Johansson 2015; \\
\hline & & $T B L 1 Y^{6}$ & Prostate, Kidney (cortex), Pancreas, Oesophagus, Thyroid and Adipose & $\begin{array}{l}\text { Maan et al. 2017) } \\
\text { (Maan et al. 2017) }\end{array}$ \\
\hline & & TSPY $4^{8} / 8^{8} / 3^{8} / 1^{7} / 10^{8}$ & Presumed to be expressed on Testis & (Jangravi et al. 2013) \\
\hline \multirow{2}{*}{ Centromere } & Yp11.1 & Heterochromatin & & (Jangravi et al. 2013; \\
\hline & Yq11.1 & несетосптотыа & & Kujawski et al. 2004) \\
\hline \multirow[t]{37}{*}{$Y q$} & Yq11.21 & $\underline{U^{\prime} P g Y^{5}}\left(=D F F R Y^{2}\right)$ & Ubiquitously expressed ${ }^{\mathrm{a}}$ & (Maan et al. 2017) \\
\hline & & $D D X 3 Y^{7}\left(=D B Y^{3}\right)$ & Ubiquitously expressed & (Maan et al. 2017) \\
\hline & Yq11.221 & $U T Y^{3}$ & Ubiquitously expressed ${ }^{a}$ & (Maan et al. 2017) \\
\hline & & TMSB4Y $Y^{6}\left(=T B 4 Y^{3}\right)$ & Ubiquitously expressed ${ }^{a}$ & (Maan et al. 2017) \\
\hline & & VCY1B & Testis & (Maan et al. 2017) \\
\hline & & $\overline{V C Y 1^{4}}$ & Testis & (Kido and Lau 2015) \\
\hline & & NLGN4Y $Y^{6}$ & Ubiquitously expressed ${ }^{a}$ & (Maan et al. 2017) \\
\hline & Yq11.222 & $\underline{X K R Y^{1}}$ & Testis specific & (Kido and Lau 2015) \\
\hline & & $\overline{C D Y 2 B^{7}}$ & Testis & (Maan et al. 2017) \\
\hline & & $\overline{C D Y 2 A^{7}}$ & Testis specific & (Kido and Lau 2015) \\
\hline & & $X K R Y 2^{7}$ & Testis specific & (Kido and Lau 2015) \\
\hline & & $\underline{\text { HSFY1 }^{7}}$ & $\begin{array}{l}\text { Testis, Prostate, Adrenal Gland, Coronary Artery, Thyroid, Brain (Cortex } \\
\text { and Cerebellum) }\end{array}$ & (Maan et al. 2017) \\
\hline & & $\mathrm{HSFY}^{7}$ & Testis specific & (Kido and Lau 2015) \\
\hline & & $K D M 5 D^{7}\left(=S M C Y^{1}\right)$ & Ubiquitously expressed ${ }^{a}$ & (Maan et al. 2017) \\
\hline & & Heterochromatin & & $\begin{array}{l}\text { (Skaletsky et al. } \\
\text { 2003) }\end{array}$ \\
\hline & Yq11.223 & EIF1AY3 & Ubiquitously expressed ${ }^{a}$ & (Maan et al. 2017) \\
\hline & & $R_{R S 4 Y 2^{6}}$ & Ubiquitous expression & (Kido and Lau 2015) \\
\hline & & $\underline{P R O R Y^{8}}\left(=C Y o r f 17^{7}\right)$ & Testis & $\begin{array}{l}\text { (Kido and Lau 2015; } \\
\text { Maan et al. 2017) }\end{array}$ \\
\hline & & $R B M Y 1 B^{8}$ & Testis & (Kido and Lau 2015) \\
\hline & & $R B M Y 1 A 1^{7}$ & Testis specific & (Kido and Lau 2015) \\
\hline & & $\overline{R B M Y 1 D^{7}}$ & Testis specific & (Kido and Lau 2015) \\
\hline & & $\overline{R B M Y 1 E^{8}}$ & Testis specific & (Kido and Lau 2015) \\
\hline & & $\underline{P R Y 2^{7}}$ & Testis, Heart, Lung, White blood cells & (Kido and Lau 2015) \\
\hline & & ${ }_{R B M Y} F^{7}$ & Testis specific & $\begin{array}{l}\text { (Jangravi et al. 2013; } \\
\text { Kido and Lau 2015) }\end{array}$ \\
\hline & & $\underline{R B M Y 1 J^{7}}$ & Testis specific & (Maan et al. 2017) \\
\hline & & $\underline{P R Y^{3}}$ & Testis & (Maan et al. 2017) \\
\hline & & $B P Y 2^{3}$ & Testis & (Maan et al. 2017) \\
\hline & & $\overline{D A Z 1^{7}}$ & Testis, Stomach and Liver & (Maan et al. 2017) \\
\hline & & $\overline{D A Z 2^{7}}\left(=S P G Y^{1}\right)$ & Predominantly in Testis ${ }^{b}$ & (Kido and Lau 2015) \\
\hline & & $C D Y 1 B^{7}$ & Testis specific & (Kido and Lau 2015) \\
\hline & & $B P Y 2 B^{7}$ & Testis specific & (Kido and Lau 2015) \\
\hline & Yq11.23 & $\overline{D A Z 3^{7}}$ & Predominantly in Testis ${ }^{b}$ & (Kido and Lau 2015) \\
\hline & & $\overline{D A Z 4^{7}}$ & Predominantly in Testis ${ }^{b}$ & (Kido and Lau 2015) \\
\hline & & $B P Y 2 C^{7}$ & Testis specific & (Kido and Lau 2015) \\
\hline & & $\overline{C D Y 1 A^{8}}$ & Testis specific & (Kido and Lau 2015) \\
\hline & Yq12 & Heterochromatin & & $\begin{array}{l}\text { (Jangravi et al. 2013; } \\
\text { Skaletsky et al. 2003) }\end{array}$ \\
\hline & & PAR2 & & $\begin{array}{l}\text { (Jangravi et al. 2013; } \\
\text { Skaletsky et al. 2003) }\end{array}$ \\
\hline
\end{tabular}


include the TSPY repeats ( $~ 35$ copies; this varies among individuals), 3 inverted repeats (IR1, IR2, and IR3), 2 arrays of no-long-open-reading-frame clusters (NORF; including TTTY1, 2, 6, 7, 8, 18, 19,21, and 22), and 8 palindromes (corresponding to $25 \%$ of euchromatin) [Skaletsky et al., 2003].

The heterochromatin of the $\mathrm{Y}$ chromosome is approximately $30 \mathrm{Mb}$ in length, and it is highly abundant in Alu and LINE repetitive sequences, elements that are transposable in the genome [Lander et al., 2001; Skaletsky et al., 2003]. These transposons make a characteristic signature on the chromosome that is passed from one generation to the other [Butler, 2012].

\section{Y Chromosome Function and Disease Studies}

In addition to its contribution to male gonad development during embryogenesis, the $\mathrm{Y}$ chromosome is responsible for fertility maintenance and Y-linked phenotypic male traits [Affara et al., 1996; Jobling and TylerSmith, 2000]. It may also contribute to other functions, such as skeleton growth, tooth size, handedness, and cerebral asymmetry [Crow, 2002; Ginalski et al., 2004; Singh et al., 2011]. A study performed by Jangravi et al. [2013] established that MSY proteins have roles in several biological processes including transcription, cell differentiation, metabolic processes, tissue development, chromatin modification, protein translation, sex differentiation, cell adhesion, and cell proliferation, among others. For example, TSPY has functions in the renewal of spermatogonia. This gene is also speculated to be involved in meiotic cycle regulation and is even considered to be a proto-oncogene [Lau et al., 2011; Lau et al., 2019]. Another important Y-chromosomal gene is $S R Y$, which was reported by Yuan et al. [2001] to be able to interact with the androgen receptor and negatively regulate its activity.

As expected, the $\mathrm{Y}$ chromosome has been the target of several studies regarding Y-linked diseases. Male infertility is frequently associated with microdeletions in the azoospermia factor locus of the Y chromosome, highlighting the importance of this chromosome in male spermatogenesis [Batiha et al., 2018]. Diseases concerning sexual development include Y chromosome gonadal dysgenesis (Y-GD) which is characterized by underdevelopment of the testis [Berberoglu et al., 2018]. In Y-GD, 2 groups can be distinguished: 46,XY females (Swyer syndrome) and 45,X/46,XY mosaicism [Berberoglu et al., 2018]. Additionally, Y-GD patients frequently present gonadoblastoma, a benign tumour that has been pro- posed to have an associated locus near the centromere of the Y chromosome [Lau et al., 2011; Knauer-Fischer et al., 2015].

Furthermore, viral infections and cardiovascular diseases such as hypertension, coronary artery disease, myocardial infarction, and stroke have been associated with the Y chromosome [Case and Teuscher, 2015; Johansson, 2015]. For instance, high blood pressure has been linked to this chromosome in spontaneously hypertensive rats (SHR) and stroke-prone spontaneously hypertensive rats (SHRSP) [Ely and Turner, 1990; Davidson et al., 1995; Kren et al., 2001]. Ely and Turner [1990] demonstrated for the first time the influence of the $\mathrm{Y}$ chromosome in blood pressure regulation. These authors transferred the Y chromosome of SHR to WKY (normotensive) rats, which resulted in an increase of 12 $\mathrm{mmHg}$ in systolic blood pressure in the WKY rat. In SHR rats, Sry is a gene complex, which includes 7 different $S r y$ copies, Sry1, Sry2, Sry3, Sry3A, Sry3B, Sry3B1, and Sry3C, each producing a functional protein, and these proteins are extremely similar to each other [Ely et al., 2011]. Although in different tissues expression levels of the different copies vary, all copies are expressed in the respective tissues, and all are able to produce a SRY protein [Ely et al., 2011]. For instance, SRY1 is capable of elevating blood pressure by increasing sympathetic nervous system activity in rats. SRY1 interacts with tyrosine hydroxylase, rising its levels and norepinephrine's levels as well, which leads to a higher blood pressure [Milsted et al., 2010]. On the other hand, the Sry $3 A$ gene, which is exclusively located on the SHR Y chromosome, was introduced in the WKY rat, and its protein increased renal sodium reabsorption by $50 \%$ in Sry3A-overexpressing WKY rats [Ely et al., 2011]. The authors suggested that this effect may be explained by an increase in renal angiotensin II (Ang-II) [Ely et al., 2011]. In fact, when Sry1, Sry2, and Sry3 expression vectors were co-transfected into cultured hamster cells, SRY3 was found to upregulate the activity of the angiotensin, renin, and $A C E$ gene promoters and to downregulate the activity of the ACE2 promoter [Milsted et al., 2010]. The proteins encoded by these genes are part of the renin-angiotensin system (RAS), and the main RAS products that regulate blood pressure are Ang-II (raises blood pressure) and Ang-(17) (decreases blood pressure) [Milsted et al., 2010]. The upregulation of angiotensin, renin, and $A C E$ genes (responsible for raising Ang-II levels) and the downregulation of ACE2 genes (responsible for raising Ang-(1-7) levels) increase blood pressure [Milsted et al., 2010]. Therefore, the Y chromosome has also been proposed to 
be a contributor to increased blood pressure in male rodent models of hypertension [Milsted et al., 2010; Ely et al., 2011; Prokop et al., 2016].

Since the Y chromosome is passed on exclusively by paternal lineages, most studies explore different $Y$ haplogroups which are defined by specific genetic variants occurring in the MSY region of the $\mathrm{Y}$ chromosome. The major Y haplogroups are A\&B, CT, C, D, E, F, G, H, I, J, K, L\&T, K2, K2a, K2a1, K2b1, NO \& NO1, N, O, M\&S, P, $\mathrm{Q}$, and R. Several studies have already evaluated the association of different $Y$ haplogroups with diseases. Y haplogroup I is the most reported example. Representing one-fifth of the European population, this Y haplogroup has been associated with a faster human immunodeficiency virus (HIV) progression, a higher resistance to highly active antiretroviral therapy (HAART) in HIV patients, and with coronary artery disease (CAD). Y haplogroup I subjects show differences in macrophage expression of inflammation and immune-related genes, which may lead to altered immune responses and a predisposition to CAD, rapid HIV progression and an increased resistance to HAART [Charchar et al., 2012; Maan et al., 2017]. Moreover, variants of Y haplogroup K (southwest Asia) have been associated with a more than 2 -fold increased risk of atherosclerotic plaque occurrence in the carotid and femoral artery bifurcations, but an explanation for this association remains to be elucidated [Hiura et al., 2008]. Another synergic explanation for the associations between the $\mathrm{Y}$ chromosome and disease is the HindIII polymorphism in the Y chromosome [Charchar et al., 2004]. This polymorphism has been associated with cholesterol levels, namely higher LDL levels, mainly in young men that had a father with myocardial infarction history [Charchar et al., 2004]. However, since these findings were observed in younger subjects, these associations must be confirmed by using functional studies focusing on the Y chromosome's influence on cholesterol levels in adult men.

Additionally, the Y chromosome has also been studied in association with cancer, namely prostate cancer (PC). Paracchini et al. [2003] reported that, in the Japanese population, Y haplogroup O3 was responsible for a higher predisposition to PC. Moreover, another study in the Japanese population demonstrated an increased risk of PC in Y haplogroup DE when compared with O2b and O2b1 [Ewis et al., 2006]. According to the authors, these findings can explain the low PC incidence rate in the Japanese population. On the other hand, Plaseska Karanfilska et al. [2009] observed an increased PC frequency in Y haplogroup R1a in comparison with the controls. The hy-

Loss of Chromosome Y Implications on

Health and Forensic Sciences pothesis that an altered $S R Y$ expression could interfere with androgen receptor activity in PC was suggested as a possible explanation for $\mathrm{Y}$ haplogroup associations with this disease [Paracchini et al., 2003]. Other Y chromosome-related alterations in PC include loss of the $\mathrm{Y}$ chromosome which is the most reported aberration in this type of cancer [Johansson, 2015]. Indeed, in a study on athymic nude mice, the tumorigenicity of the PC-3 cell line, a human PC cell line lacking a Y chromosome, was suppressed when an exogenous Y chromosome was added [Vijayakumar et al., 2005]. These findings also suggest the existence of tumour suppressor genes on this sex chromosome [Vijayakumar et al., 2005].

The fact that the Y chromosome has been studied in association with different types of diseases proposes that this chromosome may contribute to the lifespan differences observed between men and women [Forsberg, 2017; Forsberg et al., 2017].

\section{Forensic Applications of the Y Chromosome}

The Y chromosome is a paternal linage marker, and it is extensively used in forensic sciences in different analyses. The genetic information of this chromosome is used for evidence analysis, paternity testing, anthropology investigations, human migration pattern studies, and genealogical research [Butler, 2012].

Forensic applications of the Y chromosome are mainly based on the analysis of genetic polymorphisms such as short tandem repeats (STRs) and single nucleotide polymorphisms (SNPs) [Kayser, 2017]. STRs are polymorphic individualizers that are dispersed all over the genome. They consist in the repeat of repeat units ranging from 2 to 6 bp in length [El-Alfy and Abd El-Hafez, 2012]. On the other hand, SNPs are genetic variations of a single nucleotide, and they occur on average in every $300 \mathrm{bp}$ throughout the genome [Sobrino et al., 2005]. Every single person has a different combination of autosomal STRs and SNPs that makes his/her own fingerprint, allowing human differentiation and individualization [Kayser, 2017]. Y chromosome-specific polymorphisms, Y-STRs and Y-SNPs, are already being included in forensic commercial kits when autosomal polymorphisms are not sufficient. Actually, 27 Y-STRs are already validated, allowing paternal lineage characterization with a high degree of reliability [Gopinath et al., 2016]. In addition to that, the combination of different commercial kits and supplementary multiplexes currently allows the amplification of more than 40 Y-STR sequences [Roewer, 2019]. 
In forensic science, utilization of the $\mathrm{Y}$ chromosome brings several advantages. This chromosome is exclusively characteristic of males, and it is crucial to identify males in forensic cases of DNA sample mixtures (female and male DNA) [Prinz and Sansone, 2001]. Moreover, since $95 \%$ of this chromosome does not recombine, there is a uniparental transmission of the $\mathrm{Y}$ chromosome, from father to son, as a haplotype (entire chromosome block) [Butler, 2012]. These characteristics allow the application of the Y chromosome in forensic matters which is extremely useful.

Based on polymorphisms, 2 categories, biallelic and multiallelic, arise when the Y chromosome is studied, defining haplogroups and haplotypes, respectively [Butler, 2012]. Haplogroups can be defined using mainly Y-SNPs and Alu elements that have lower mutations rates $\left(\sim 10^{-8}\right.$ to $10^{-9}$ per generation) than Y-STRs [Hammer, 1994]. Whereas haplotypes are mainly characterized based on Y-STRs and minisatellites (variable number of tandem repeats, VNTRs; $10-15 \mathrm{bp}$ ) that have higher mutation rates than Y-SNPs [de Knijff, 2000]. These categories have different applications: Y haplogroups are used to study human migration and biogeographic ancestry, that is, research about the origins of a person's paternal ancestors [Phillips, 2015; Kayser, 2017]. In contrast, Y-haplotypes are more similar to a "family fingerprint," assisting in paternity testing. For example, Y-STRs are extremely useful when the father is missing or not available for autosomal STR testing, and so, a male relative (like a grandfather) can be tested instead [Butler, 2012]. If the Y haplotypes match, it can be assumed that the child belongs to the family of the missing father [Butler, 2012]. Nevertheless, it is important to notice that the non-recombination on the majority of the Y chromosome also decreases the discrimination power among related family individuals [Butler, 2012]. So, Y haplotypes are not sufficient to distinguish between male individuals of the same family linage. Therefore, as was mentioned before, Y-STRs are only studied when autosomal STRs alone cannot answer law court's questions [Butler, 2012].

Besides polymorphisms, genes from the Y chromosome are used for sex typing of forensic samples [Butler and Li, 2014]. Most commercial kits use the amelogenin $(A M E L)$ gene along with a wide number of autosomal STRs for additional DNA profiling [Butler and Li, 2014]. The AMEL locus has 2 homologous genes located on both the $\mathrm{X}(A M E L X)$ and $\mathrm{Y}$ chromosome (AMELY) which differ by $6 \mathrm{bp}$ from each other [Mannucci et al., 1994]. This difference allows the determination of a sample's chromosomal sex [Mannucci et al., 1994]. De- spite the worldwide use of $A M E L$ fragments, several cases of failure have been reported due to AMELY deletions which have a frequency of $0.018-8.0 \%$, depending on the population [Butler and $\mathrm{Li}, 2014$ ]. To overcome $A M E L Y$ detection failures, other genes, namely $S R Y$ and TSPY are used [Tozzo et al., 2013; Butler and Li, 2014]. Moreover, due to the main involvement of $S R Y$ in gonadal genesis and TSPY's association with spermatogenesis, these novel sex typing markers allow for a more accurate chromosomal sex determination [Morikawa et al., 2011]. The inclusion of these markers in commercial forensic kits is crucial to avoid erroneous sex sample determination due to deletion of $A M E L Y$.

\section{Loss of Chromosome $Y$}

LOY in leucocytes of aging men was first reported by Jacobs et al. [1963]. Furthermore, in 1995, an in situ hybridization study on healthy males, aged between 1 week and 93 years, demonstrated an age-dependent loss of the $\mathrm{Y}$ chromosome in donors older than 16 years [Guttenbach et al., 1995]. LOY classification does not include Y nullisomy, since nullisomy is a result of nondisjunction during meiosis and, thus, does not act as a mosaic event, but affects all postzygotic cells [Forsberg, 2017; Forsberg et al., 2017]. Other aberrations such as microdeletions, observed, for example, in male infertility, are not considered LOY either [Dumanski et al., 2017].

The mechanism by which LOY occurs is yet to be elucidated. It is not known whether the $\mathrm{Y}$ chromosome is lost as a whole or if it is a gradual deletion process during the lifespan. Recently, a sequencing coverage of 20 MSY-specific genes showed that LOY samples had attenuated amplification of all of the Y chromosome-specific loci [Arseneault et al., 2017]. This evidence correlates with the assumption that the loss of this chromosome occurs as a whole. Based on data gathered until now, some hypotheses of how LOY occurs in human cells can be formulated. The first one is based on LOY being a neutral event happening due to environment triggers that lead to missegregation in mitosis [Dumanski et al., 2015]. The second hypothesis resides on aging itself: when telomeric shortening increases chromosomal instability, it promotes chromosomal degradation and, eventually, Y chromosomal loss in elderly males [Guttenbach et al., 1995]. Anaphase shortening favouring accidental losses is the third hypothesis; this is due to the tendency of Y-chromosomal replication to be performed in a late stage of Sphase [Persani et al., 2012]. 
The first study to report LOY associated with disease was published in 1985 [Holmes et al., 1985]. In this study, acute myeloid leukaemia patients exhibited Y chromosome hypoploidy in the bone marrow [Holmes et al., 1985]. Since then, several reports demonstrated an potential association between LOY and the development of diseases such as Alzheimer, cardiovascular events, autoimmune diseases, and a wide number of cancers [Holmes et al., 1985; Castedo et al., 1992; Hunter et al., 1993; Guttenbach et al., 1995; Sauter et al., 1995; Brunelli et al., 2003; Gilgenkrantz, 2008; Klatte et al., 2009; Persani et al., 2012; Lleo et al., 2013; Dumanski et al., 2016; Noveski et al., 2016; Forsberg, 2017; Forsberg et al., 2017; Haitjema et al., 2017; Hirata et al., 2018]. Furthermore, Arseneault et al. [2017] accessed a gene expression RNA-Seq dataset in male samples of normal and clear cell renal cell carcinoma. The findings correlated with lower expression of 11 Y-chromosomal genes, including KDM5D, an epigenetic modifier whose deficiency is involved in the progression of clear cell renal cell carcinoma [Arseneault et al., 2017]. The fact that LOY chromosome content seems to provide an advantage to cell proliferation may contribute to the development of diseases [Forsberg, 2017; Forsberg et al., 2017]. When LOY is present, it interacts with pathways for apoptosis and cancer [Wright et al., 2017]. Indeed, several genes that encode regulation factors of the 3 cell cycle checkpoints were found to have LOY-associated genetic variants [Wright et al., 2017]. These genetic variants negatively affected error repairing and increased cell cycle progression [Wright et al., 2017]. Other mechanisms that could link LOY to increased risk of disease include increased genome instability and possible negative effects in leucocyte immune function [Loftfield et al., 2019]. However, functional studies centred in the immune system are needed to verify if LOY can have an influence on immune function.

\section{LOY as a Biomarker}

LOY has been suggested as a possible biomarker for increased risk of disease and mortality in aging men [Forsberg, 2017; Forsberg et al., 2017a; Loftfield et al., 2018]. A biomarker is a molecule which can be easily measured in the body and itself or its products can influence or predict the incidence or the outcome of a disease [Strimbu and Tavel, 2010]. LOY has been studied in association with different types of diseases since its percentage increases over time in healthy individuals, and it has a significant increase when disease is present [Forsberg,

Loss of Chromosome Y Implications on

Health and Forensic Sciences
2017; Forsberg et al., 2017; Loftfield et al., 2018]. Interestingly, exposures to outdoor pollution and smoking also have an influence on LOY and, since these factors increase its frequency, they consequently increase the mortality risk [Dumanski et al., 2015; Wong et al., 2018]. Additionally, presence of LOY may alert the beginning of a pathological process, such as colorectal cancer and PC, as was reported by Noveski et al. [2016]. In another type of cancer, familial TGCT, LOY was significantly increased in those patients when compared with cancer-free individuals [Machiela et al., 2017]. In addition to that, healthy individuals younger than 50 years old had low frequencies of LOY in comparison to TGCT patients [Forsberg et al., 2014]. This evidence suggests that LOY could be applied as a biomarker of carcinogenesis and cancer aggressiveness [Forsberg et al., 2014]. In a study performed in patients with head and neck carcinoma, LOY was also proposed to be a poor prognosis predictor, and LOY could even increase therapy resistance in these patients [Hollows et al., 2019]. On the other hand, there are different percentages of LOY among different diseases, which suggests that LOY frequency could guide towards a more personalised diagnosis and/or treatment [Silva Veiga et al., 2012; Dumanski et al., 2016, 2017; Arseneault et al., 2017; Haitjema et al., 2017]. Recent reports have provided new insights into LOY's heritability hypothesis and which type of blood cells are more affected by LOY-associated variants [Dumanski et al., 2019; Terao et al., 2019; Thompson et al., 2019]. Dumanski et al. [2019] observed that LOY frequency was considerably higher in myeloid lineage cells than in lymphoid lineage cells, while Thompson et al. [2019] found that haematopoietic stem progenitor cells were most strongly affected by LOY-associated gene variants. Genome-wide association studies performed by Terao et al. [2019] demonstrated a polygenic architecture and strong heritability enrichment in regions which are near genes that are specifically expressed in multipotent progenitor cells. Furthermore, the observations of Thompson et al. [2019] suggest that genetic variants associated with LOY affect 3 different temporal modes in the haematopoiesis tree: haematopoietic stem cells, multipotent progenitor cells, and common myeloid progenitor cells [Thompson et al., 2019]. Therefore, LOY may be reflecting the genomic instability occurring in other cells and tissues [Thompson et al., 2019; Grassmann et al., 2020]. Consequently, the use of LOY as a biomarker has the potential to improve the prevention, detection, monitorization, and prognosis of different diseases.

In addition to disease risk, LOY can also be of use in the determination of an individual's biological age. Bio- 
logical age is a reflexion of the aging state that evaluates the health status, and a quantification based in numbers cannot be given [Kang et al., 2018]. Actually, biological age is more a predictor of lifespan than a predictor of the chronological age (time that has passed since birth) [Jylhävä et al., 2017]. The frequency of LOY is age-related; males under 50 years old have low LOY frequencies contrasting with the fast increase in the percentage of LOY by the age of 80 and beyond [Loftfield et al., 2019]. A biomarker of biological age has the potential to improve the evaluation of a person's health status and lifespan [Kang et al., 2018]. LOY as a biological age biomarker could be applied in work damage evaluation. For instance, LOY could be helpful to prove the negative effects of the exposure to a certain work/professional factor that could reduce the employee's lifespan. Nowadays, the use of biological age markers is becoming very useful for insurance companies [Chang, 2009; Kang et al., 2018]. The client's lifespan is already used to decide what type of life insurance and what prices are going to be applied to a specific person [Chang, 2009; Kang et al., 2018]. This brings up privacy issues, and there is a need to increase government regulations that concern biological age use to assure that human rights remain protected. The use of LOY, as a resource to access biological age, would benefit both health care and forensic fields.

LOY could be of further use in forensic psychology, in which Kimura et al. [2018] suggested an association between LOY and the higher rate of completed suicide that predominates in the male population. Their study compared LOY found in post mortem (after death) blood samples and brain samples of both controls and suicide completers. The latter had abnormal LOY rates beyond the normal age-dependent loss [Kimura et al., 2018].

Considering the implications of LOY in forensics, they are yet to be clarified. Y-STRs are used in paternity testing and other forensic cases; if the $\mathrm{Y}$ chromosome is missing, this could mean that less Y-STR or Y-SNP content is detected. The fact that an Y-STR locus could be missing has been described as Y-STR allelic dropout, this phenomenon does not occur in high frequency but can alter the signal strength of the analysis when present [Andersen et al., 2013]. Therefore, forensic work can be compromised by LOY by its interference with the accuracy of sample analysis. There are no studies on LOY in relation to YSTR or Y-SNP allelic dropout. However, other aberrations, such as microdeletions and their associations with Y-STR allelic dropout are starting to be explored [Wang et al., 2019].
On the other hand, blood and buccal samples are the most frequently used sources of information when a new forensic case appears [Shewale and Liu, 2016]. Some authors have reported the presence of LOY in buccal cells as well [Jacobs et al., 2012; Zhou et al., 2016; Forsberg et al., 2019]. Since these 2 types of biological samples are routinely used in forensic laboratories, LOY could introduce biased results in routine procedures. Usually, DNA is extracted from biological samples and Y-STRs are analysed by capillary electrophoresis. If LOY affects the male individual's buccal or blood cells, it is possible that, during DNA analyses of biological samples, the $\mathrm{Y}$ chromosome is missing. To prove in court that this particular male individual was in the crime scene then becomes more difficult. In cases of sexual assault, where a man raped a woman, the crucial evidence that distinguishes the mixture of samples and proves that a man was in the crime scene, is the Y chromosome [Campos et al., 2014]. However, it is important to notice that, in sexual assault, the evidence that predominates in the victim's clothes or vaginal samples are sperm residuals, in which the $\mathrm{Y}$ chromosome is usually well identified. LOY occurs during mitosis; since sperm cells arise from meiosis, it is not expected to find LOY in this type of cells. In fact, nullisomy of the Y chromosome could occur instead, since this event occurs only during meiotic division. Giving the importance of the $\mathrm{Y}$ chromosome in forensic case solving, it is crucial to identify if LOY could be responsible for the introduction of biased results during other types of forensic sample analysis.

Different methods have been used to identify and quantify LOY. At first, LOY was detected by karyotype analysis methods [Jacobs et al., 1963; Holmes et al., 1985; Castedo et al., 1992; Klatte et al., 2009]. Technology advancement brought in situ hybridization which became the most common technique to identify LOY [Jacobs et al., 1963; Hunter et al., 1993; Guttenbach et al., 1995; Sauter et al., 1995; Kujawski et al., 2004; Al-Saleem et al., 2005; Silva Veiga et al., 2012; Lleo et al., 2013]. Recently, more sophisticated methods emerged to detect LOY in biological samples. For instance, SNP-array data analysis uses MSY-specific probes, which allow a continuous estimation of LOY in a DNA sample [Forsberg et al., 2014, 2017; Dumanski et al., 2015, 2016; Zhou et al., 2016; Forsberg, 2017; Haitjema et al., 2017]. However, bad quality SNP-array data can lead to bias in LOY estimation; thus, accessing the SNP-array quality at the sample level is also required [Dumanski et al., 2016]. Another method that can be used to detect LOY is whole-genome sequencing 
(WGS) [Danielsson et al., 2020]. This method compares the read depth on the $\mathrm{Y}$ chromosome in relation to the full human genome. The copy number of the male sex chromosome can be estimated by using read counts, resulting in a median ploidy of the Y [Danielsson et al., 2020].

Alternative to the expensiveness of the previously mentioned methods, and the increased amount of data that need to be interpreted in SNP-arrays and WGS, PCRbased methods can be a better standardization option. For example, several studies have used a Y/X ratio to detect LOY [Noveski et al., 2016; Hirata et al., 2018; Kimura et al., 2018]. Multiplex quantitative fluorescent-PCR amplifies the 2 AMEL fragments that are on both the $\mathrm{X}$ and $\mathrm{Y}$ chromosomes. The products are quantified by capillary electrophoresis, and the results are then compared with mixed samples for the assessment of LOY [Noveski et al., 2016; Hirata et al., 2018; Kimura et al., 2018]. The mixed samples have different proportions of a normal XY male and from an individual with Turner syndrome $(45, \mathrm{X})$. These proportions mimic different percentages of LOY, and the analysed samples are compared with these proportions to estimate LOY [Noveski et al., 2016; Hirata et al., 2018; Kimura et al., 2018]. On the other hand, Danielsson et al. [2020] reported the use of droplet digital PCR (ddPCR) to identify LOY. This method is also based on AMELY/AMELX ratio, but differs in the resources used to achieve this ratio. ddPCR is a TaqMan-based method used to relatively quantify the number of $\mathrm{Y}$ and $\mathrm{X}$ chromosomes in a given sample [Danielsson et al., 2020]. DNA is digested with HindIII enzyme, diluted with ionized water, and mixed with all the needed elements for PCR amplification. This mixture is introduced in a droplet generator and analysed by a droplet reader in which the Y/X ratio readings are collected [Danielsson et al., 2020]. Nevertheless, it is important to consider that there is a low frequency of $A M E L Y$ deletion in men, ranging from 0.018 to $8.0 \%$, depending on the population [Butler and Li, 2014]. Even though this deletion is not that frequent, it may interfere with AMELY/AMELX ratio readings and promote false positives for LOY. Thus, the possible interference of $A M E L Y$ deletion must be further studied.

Another method that, to our knowledge, has not yet been used to identify LOY is a relative quantification of the presence of the Y chromosome using real-time PCR (qPCR). qPCR is a specific, fast, sensible, and less expensive method [Schmittgen and Livak, 2008]. This type of PCR is based on a quantitative endpoint, cycle threshold $(\mathrm{Ct})$, which is the moment when the equip- ment detects fluorescence, and in that moment the amplification of the targeted gene begins [Schmittgen and Livak, 2008]. The Ct value is inversely related to the amount of the amplification product; thus, the bigger the $\mathrm{Ct}$, the lower is the amount of amplicon in the reaction [Schmittgen and Livak, 2008]. This method has the potential to become a way of relatively measuring the presence of LOY using a constitutive gene of the Y chromosome, such as $S R Y$, and another gene as an endogenous control. This way, the $\mathrm{Ct}$ of both endogenous and $S R Y$ gene could be measured and compared, the difference between them would allow the identification of LOY in a short-time procedure.

Besides identification methods, there is a need to quantify the percentage of cells that no longer have the $Y$ chromosome. Until now, 3 possible methods have been suggested to estimate the percentage of LOY in a given sample. Forsberg et al. [2014] first tried to translate SNParray data into percentage of LOY. Data were analysed using median Log $\mathrm{R}$ ratio of SNP-probes in the MSY (mLRR-Y), and depending on the analysed data, mLLR-Y thresholds were created. For example, samples with mLLR-Y readings below -0.139 and -0.40 were estimated to have more than 18 and 35\% cells affected by LOY, respectively [Forsberg et al., 2014]. Although this was the first approach, 2 further formulas were established. Grassmann et al. [2020] developed a formula based on previously reported chip genotyping data and karyotyping data to estimate the percentage of LOY: percentage of cells with $\mathrm{LOY}=1.8 \times\left(1-2^{2 \mathrm{mLRR}-\mathrm{Y}}\right)$. While Danielssonn et al. [2020] developed a formula for LOY estimation that strongly correlated with data retrieved from 3 different methods (SNP-array, WGS, and ddPCR): percentage of cells with LOY $=100 \times\left(1-2^{2 \mathrm{mLRR}-\mathrm{Y}}\right)$. However, considering the different methods, different population characteristics, and different biological fluid used for DNA analysis, a standard method that can both identify and quantify LOY is yet to be developed.

There is the speculation that LOY occurs as a loss of the whole chromosome event. However, Heller et al. [1996] showed that even if the Y chromosome was blasted apart, parts (e.g., p or q arm) might be replicated and passed on to daughter cells. Since the hypothesis of the $Y$ chromosome being lost as a whole is yet to be confirmed, LOY quantification methods may only search for a part of the chromosome that has not been lost. The findings of Heller and collaborators may explain variations of LOY that are found among different diseases and quantification methods. 


\section{Conclusion and Future Perspectives}

The potential applications of LOY as a biomarker must be validated. Different percentages of LOY are observed in different types of diseases [Silva Veiga et al., 2012; Dumanski et al., 2016, 2017; Arseneault et al., 2017; Haitjema et al., 2017]. For instance, TGCT affects mainly individuals aged between 15 and 40 years, and a higher degree of LOY can be detected in these cancer patients [Machiela et al., 2017]. Since LOY is present at a lower degree in healthy individuals mainly before 50 years old, the substantial increase of LOY in TGCT individuals could allow this biomarker to help in TGCT early detection. However, it is important to note that LOY associations with disease considerably vary among different studies. This could be due to sample size effects, since most of the authors cited in this review had small sample sizes [Holmes et al., 1985; Castedo et al., 1992; Hunter et al., 1993; Guttenbach et al., 1995; Sauter et al., 1995; Brunelli et al., 2003; Klatte et al., 2009; Persani et al., 2012; Silva Veiga et al., 2012; Lleo et al., 2013; Arseneault et al., 2017; Haitjema et al., 2017; Machiela et al., 2017; Hirata et al., 2018]. Since larger samples are a better representation of the general population, future studies should consider sample size as well as randomized selection as an important factor to take further conclusions regarding LOY and the way to improve current prediction algorithms. Also, the characterization of LOY in each disease would improve preventive measures in health care.

Establishing if LOY affects Y-SNP and Y-STR analyses is crucial to understand if LOY can promote false-negatives, other types of errors or missing evidence essential to solve forensic cases. LOY can also be detected in buccal mucosa and in post mortem samples. Since the analysis was conducted in dead persons, more research on living individuals must be developed to avoid sample degradation. In addition to that, since blood and buccal samples are routinely used in forensic cases, the study of the influence of LOY on the forensic analyses of these samples must be clarified. The further reinforcement of research by testing samples with LOY with different Y-STR kits is crucial to understand in which way this loss may impact forensic case solving.

Despite the disadvantages that LOY may provide to forensics, its association with aging could be an important contribution to help establishing a sample's biological age. The use of LOY as a biological age biomarker has applications in both health and forensics. Since LOY can be helpful in the development of a health index, longterm studies validating the use of this biomarker should demonstrate its potential in assisting in the evaluation of one's lifespan and health status. Moreover, the use of biological age to categorize which kind of life insurance a person is going to get is a reality which is closer than we think. The development and implementation of a tight regulation on this subject in each country would benefit the clients, preserving their human rights. On the other hand, LOY could be of use to access the effects of work environment factors in a worker's lifespan. Since there are environment factors which increase LOY percentage, this biomarker of biological age would help workers prove that their health has become worse due to their exposure to a certain work factor.

Currently, there is no standard method for LOY detection and quantification, and literature lacks a comparative study of the different methods mentioned in this review. Since research on LOY has increased dramatically over the last decade, the development of a single standard method to access the percentage of LOY would help to better understand LOY among different populations. Even though there are already 2 possible algorithms to estimate the percentage of LOY, the authors mention that they present some limitations. These obstacles need to be surpassed in order to achieve a standard method appropriate for any population. Therefore, standardization is the next step which will allow comparison between studies, so more accurate conclusions can be accomplished in future research.

Although most $Y$ chromosome genes have been identified, their functions and interactions outside the male reproductive system must be better understood. The knowledge of their function will certainly help to understand the current correlation of LOY to early deaths in male individuals as well as with several diseases. For instance, Y chromosome aneuploidy models could be developed using the CRISPR/Cas9 system. There is already a study where this technology was used to successfully eliminate an entire chromosome in cell cultures, embryos, and in vivo [Zuo et al., 2017]. Further research based on LOY models would help to elucidate the mechanism by which LOY is associated with increased overall mortality risk. Recently, a critical review on the origin and consequences of the Y chromosome's absence has gathered all the available information concerning the biological origins and clinical consequences of LOY. Guo et al. [2020] highlight which pathologies have been associated with LOY, and there is an evaluation of the causal and consequence relationships that could connect this genetic aberration to pathogenic conditions. Nevertheless, functional studies that access interactions of the $\mathrm{Y}$ 
with the expression of immune system genes and LOYassociated variants in humans are needed to verify if LOY can have a depriving impact on human biological functions.

Efforts to answer questions about LOY are a step towards its implementation as biomarker that can benefit both health and forensic fields.

\section{Acknowledgement}

We would like to thank Liga Portuguesa Contra o Cancro Núcleo Regional do Norte - LPCC-NRN (Portuguese League Against Cancer-North Branch). M.M. is a recipient of a research scholarship awarded by LPCC-NRN.

\section{Disclosure Statement}

The authors have no conflicts of interest to declare.

\section{Funding Sources}

This research did not receive any specific funding from agencies in the public, commercial, or not-for-profit sectors.

\section{Author Contributions}

Conceptualization: B.B., A.L.T. Methodology and study analyses: B.B., M.M. Writing, original draft preparation: B.B. Writing, review and editing: M.M., A.L.T., R.M. Supervision: A.L.T., R.M.

\section{References}

Affara N, Bishop C, Brown W, Cooke H, Davey P, et al: Report of the Second International Workshop on Y Chromosome Mapping 1995. Cytogenet Cell Genet 73:33-76 (1996).

Al-Saleem T, Balsara BR, Liu Z, Feder M, Testa JR, et al: Renal oncocytoma with loss of chromosomes $\mathrm{Y}$ and 1 evolving to papillary carcinoma in connection with gain of chromosome 7 . Coincidence or progression? Cancer Genet Cytogenet 163:81-85 (2005).

Andersen MM, Mogensen HS, Eriksen PS, Olofsson JK, Asplund M, Morling N: Estimating YSTR allelic drop-out rates and adjusting for interlocus balances. Forensic Sci Int Genet 7: 327-336 (2013).

Arseneault M, Monlong J, Vasudev NS, Laskar RS, Safisamghabadi M, et al: Loss of chromosome $\mathrm{Y}$ leads to down regulation of KDM5D and KDM6C epigenetic modifiers in clear cell renal cell carcinoma. Sci Rep 7:44876 (2017).

Batiha O, Haifawi S, Al-Smadi M, Burghel GJ, Naber Z, et al: Molecular analysis of CAG repeat length of the androgen receptor gene and $\mathrm{Y}$ chromosome microdeletions among Jordanian azoospermic infertile males. Andrologia, Epub ahead of print (2018).

Berberoglu M, Siklar Z, Ankara University DSD Ethics Committee: The evaluation of cases with Y-chromosome gonadal dysgenesis: clinical experience over 18 years. J Clin Res Pediatr Endocrinol 10:30-37 (2018).

Brunelli M, Eble JN, Zhang S, Martignoni G, Cheng L: Gains of chromosomes 7, 17, 12, 16, and 20 and loss of $Y$ occur early in the evolution of papillary renal cell neoplasia: a fluorescent in situ hybridization study. Mod Patho 16:1053-1059 (2003)

Butler EK, Li R: Genetic markers for sex identification in forensic DNA analysis. J Forensic Invest 2:10 (2014).
Butler JM: Y-chromosome DNA testing, in Butler JM (ed): Advanced Topics in Forensic DNA Typing: Methodology, pp 371-403 (Elsevier, Amsterdam 2012).

Campos EA, Pitta DR, Costa FA, Campos VM, Yela D, Fernandes A: DNA extraction from filter-paper spots of vaginal samples collected after sexual violence. Int J Gynaecol Obstet 126:23-27 (2014).

Case LK, Teuscher C: $Y$ genetic variation and phenotypic diversity in health and disease. Biol Sex Differ 6:6 (2015).

Castedo S, Correia C, Gomes P, Seruca R, Soares $\mathrm{P}$, et al: Loss of $\mathrm{Y}$ chromosome in gastric carcinoma. Fact or artifact? Cancer Genet Cytogenet 61:39-41 (1992).

Chang CC: Adjustable Biological-Age Pricing for the Global Market (Society of Actuaries, University in Taichung, Taiwan 2009).

Charchar FJ, Tomaszewski M, Lacka B, Zakrzewski J, Zukowska-Szczechowska E, et al: Association of the human Y chromosome with cholesterol levels in the general population. Arterioscler Thromb Vasc Biol 24:308-312 (2004).

Charchar FJ, Bloomer LD, Barnes TA, Cowley MJ, Nelson CP, et al: Inheritance of coronary artery disease in men: an analysis of the role of the Y chromosome. Lancet 379:915-922 (2012).

Colaco S, Modi D: Genetics of the human Y chromosome and its association with male infertility. Reprod Biol Endocrinol 16:14 (2018).

Cooke HJ, Brown WR, Rappold GA: Hypervariable telomeric sequences from the human sex chromosomes are pseudoautosomal. Nature 317:687-692 (1985)

Crow TJ: Handedness, language lateralisation and anatomical asymmetry: relevance of protocadherin XY to hominid speciation and the aetiology of psychosis. Point of view. Br J Psychiatry 181:295-297 (2002).
Danielsson M, Halvardson J, Davies H, Moghadam BT, Mattisson J, et al: Longitudinal changes in the frequency of mosaic chromosome Y loss in peripheral blood cells of aging men varies profoundly between individuals. Eur J Hum Genet 28:349-357 (2020).

Davidson AO, Schork N, Jaques BC, Kelman AW, Sutcliffe RG, et al: Blood pressure in genetically hypertensive rats. Influence of the Y chromosome. Hypertension 26:452-459 (1995).

de Knijff P: Messages through bottlenecks: on the combined use of slow and fast evolving polymorphic markers on the human Y chromosome. Am J Hum Genet 67:1055-1061 (2000).

Dumanski JP, Rasi C, Lönn M, Davies H, Ingelsson M, et al: Mutagenesis. Smoking is associated with mosaic loss of chromosome Y. Science 347:81-83 (2015)

Dumanski JP, Lambert JC, Rasi C, Giedraitis V, Davies H, et al: Mosaic loss of chromosome $Y$ in blood is associated with Alzheimer disease. Am J Hum Genet 98:1208-1219 (2016).

Dumanski JP, Sundström J, Forsberg LA: Loss of chromosome $\mathrm{Y}$ in leukocytes and major cardiovascular events. Circ Cardiovasc Genet 10:e001820 (2017)

Dumanski JP, Halvardson J, Davies H, RychlickaBuniowska E, Mattisson J, et al: Loss of Y in leukocytes, dysregulation of autosomal immune genes and disease risks. bioRxiv 673459 (2019).

El-Alfy SH, Abd El-Hafez AF: Paternity testing and forensic DNA typing by multiplex STR analysis using ABI PRISM 310 Genetic Analyzer. J Genet Eng Biotechnol 10:101-112 (2012).

Ely DL, Turner ME: Hypertension in the spontaneously hypertensive rat is linked to the $\mathrm{Y}$ chromosome. Hypertension 16:277-281 (1990). 
Ely D, Boehme S, Dunphy G, Hart M, Chiarappa F, et al: The Sry3 Y chromosome locus elevates blood pressure and renin-angiotensin system indexes. Gend Med 8:126-138 (2011).

Ewis AA, Lee J, Naroda T, Sano T, Kagawa S, et al: Prostate cancer incidence varies among males from different $Y$-chromosome lineages. Prostate Cancer Prostatic Dis 9:303-309 (2006).

Forsberg LA: Loss of chromosome Y (LOY) in blood cells is associated with increased risk for disease and mortality in aging men. Hum Genet 136:657-663 (2017)

Forsberg LA, Rasi C, Malmqvist N, Davies H, Pasupulati S, et al: Mosaic loss of chromosome $\mathrm{Y}$ in peripheral blood is associated with shorter survival and higher risk of cancer. Nat Genet 46:624-628 (2014).

Forsberg LA, Gisselsson D, Dumanski JP: Mosaicism in health and disease - clones picking up speed. Nat Rev Genet 18:128-142 (2017).

Forsberg LA, Halvardson J, Rychlicka-Buniowska E, Danielsson M, Moghadam BT, et al: Mosaic loss of chromosome $\mathrm{Y}$ in leukocytes matters. Nat Genet 51:4-7 (2019).

Freije D, Helms C, Watson MS, Donis-Keller H: Identification of a second pseudoautosomal region near the $\mathrm{Xq}$ and $\mathrm{Yq}$ telomeres. Science 258:1784-1787 (1992).

Gilgenkrantz S: Nettie Maria Stevens (18611912) (in French). Med Sci (Paris) 24:874-878 (2008).

Ginalski K, Rychlewski L, Baker D, Grishin NV: Protein structure prediction for the male-specific region of the human Y chromosome. Proc Natl Acad Sci USA 101:2305-2310 (2004).

Gopinath S, Zhong C, Nguyen V, Ge J, Lagacé RE, et al: Developmental validation of the Yfiler ${ }^{\circledR}$ Plus PCR Amplification Kit: an enhanced YSTR multiplex for casework and database applications. Forensic Sci Int Genet 24:164-175 (2016).

Grassmann F, International AMD Genomics Consortium (IAMDGC), Weber BHF, Veitia RA: Insights into the loss of the Y chromosome with age in control individuals and in patients with age-related macular degeneration using genotyping microarray data. Hum Genet 139:401-407 (2020).

Guo X, Dai X, Zhou T, Wang H, Ni J, et al: Mosaic loss of human $\mathrm{Y}$ chromosome: what, how and why. Hum Genet 139:421-446 (2020).

Guttenbach M, Koschorz B, Bernthaler U, Grimm T, Schmid M: Sex chromosome loss and aging: in situ hybridization studies on human interphase nuclei. Am J Hum Genet 57:11431150 (1995).

Haitjema S, Kofink D, van Setten J, van der Laan SW, Schoneveld AH, et al: Loss of Y chromosome in blood is associated with major cardiovascular events during follow-up in men after carotid endarterectomy. Circ Cardiovasc Genet 10:e001544 (2017).

Hammer MF: A recent insertion of an Alu element on the $\mathrm{Y}$ chromosome is a useful marker for human population studies. Mol Biol Evol 11:749-761 (1994).
Heller R, Brown KE, Burgtorf C, Brown WR: Mini-chromosomes derived from the human Y chromosome by telomere directed chromosome breakage. Proc Natl Acad Sci USA 93: 7125-7130 (1996).

Hirata T, Hishimoto A, Otsuka I, Okazaki S, Boku $S$, et al: Investigation of chromosome $Y$ loss in men with schizophrenia. Neuropsychiatr Dis Treat 14:2115-2122 (2018).

Hiura Y, Fukushima Y, Kokubo Y, Okamura T, Goto $Y$, et al: Effects of the $Y$ chromosome on cardiovascular risk factors in Japanese men. Hypertens Res 31:1687-1694 (2008).

Hollows R, Wei W, Cazier JB, Mehanna H, Parry $\mathrm{G}$, et al: Association between loss of Y chromosome and poor prognosis in male head and neck squamous cell carcinoma. Head Neck 41:993-1006 (2019).

Holmes RI, Keating MJ, Cork A, Trujillo JM, McCredie KB, Freireich EJ: Loss of the Y chromosome in acute myelogenous leukemia: a report of 13 patients. Cancer Genet Cytogenet 17:269-278 (1985).

Hunter S, Gramlich T, Abbott K, Varma V: Y chromosome loss in esophageal carcinoma: an in situ hybridization study. Genes Chromosomes Cancer 8:172-177 (1993).

Jacobs KB, Yeager M, Zhou W, Wacholder S, Wang Z, et al: Detectable clonal mosaicism and its relationship to aging and cancer. Nat Genet 44:651-658 (2012).

Jacobs PA, Brunton M, Court Brown WM, Doll R, Goldstein H: Change of human chromosome count distributions with age: evidence for a sex difference. Nature 197:1080-1081 (1963).

Jangravi Z, Alikhani M, Arefnezhad B, Sharifi Tabar M, Taleahmad S, et al: A fresh look at the male-specific region of the human Y chromosome. J Proteome Res 12:6-22 (2013).

Jobling MA, Tyler-Smith C: New uses for new haplotypes - the human Y chromosome, disease and selection. Trends Genet 16:356-362 (2000).

Johansson MM: The human Y chromosome and its role in the developing male nervous system. Digital Comprehensive Summaries of Uppsala Dissertations from the Faculty of Science and Technology 1285, Acta Universitatis Upsaliensis, Uppsala (2015).

Jylhävä J, Pedersen NL, Hägg S: Biological age predictors. EBioMedicine 21:29-36 (2017).

Kang YG, Suh E, Lee JW, Kim DW, Cho KH, Bae CY: Biological age as a health index for mortality and major age-related disease incidence in Koreans: National Health Insurance Service - Health screening 11-year follow-up study. Clin Interv Aging 13:429-436 (2018).

Kayser M: Forensic use of Y-chromosome DNA: a general overview. Hum Genet 136:621-635 (2017).

Kido T, Lau YF: Roles of the Y chromosome genes in human cancers. Asian J Androl 17:373-380 (2015).

Kimura A, Hishimoto A, Otsuka I, Okazaki S, Boku S, et al: Loss of chromosome Y in blood, but not in brain, of suicide completers. PLoS One 13:e0190667 (2018).
Klatte T, Rao PN, de Martino M, LaRochelle J, Shuch B, et al: Cytogenetic profile predicts prognosis of patients with clear cell renal cell carcinoma. J Clin Oncol 27:746-753 (2009).

Knauer-Fischer S, Besikoglu B, Inta I, Kneppo C, Vogt PH, Bettendorf M: Analyses of gonadoblastoma Y (GBY)-locus and of Y centromere in Turner syndrome patients. Exp Clin Endocrinol Diabetes 123:61-65 (2015).

Kren V, Qi N, Krenova D, Zidek V, Sladka M, et al: Y-chromosome transfer induces changes in blood pressure and blood lipids in SHR. Hypertension 37:1147-1152 (2001).

Kujawski M, Jarmuz M, Rydzanicz M, Szukala K, Wierzbicka M, et al: Frequent chromosome Y loss in primary, second primary and metastatic squamous cell carcinomas of the head and neck region. Cancer Lett 208:95-101 (2004).

Kumar A, Sharma M: Basics of Human Andrology: A Textbook (Springer, Singapore 2017).

Lahn BT, Page DC: Functional coherence of the human Y chromosome. Science 278:675-680 (1997).

Lander ES, Linton LM, Birren B, Nusbaum C, Zody MC, et al: Initial sequencing and analysis of the human genome. Nature 409:860921 (2001).

Lau YC, Li Y, Kido T: Battle of the sexes: contrasting roles of testis-specific protein $\mathrm{Y}$-encoded (TSPY) and TSPX in human oncogenesis. Asian J Androl 21:260-269 (2019).

Lau YF, Li Y, Kido T: Role of the Y-located putative gonadoblastoma gene in human spermatogenesis. Syst Biol Reprod Med 57:27-34 (2011).

Lleo A, Oertelt-Prigione S, Bianchi I, Caliari L, Finelli P, et al: Y chromosome loss in male patients with primary biliary cirrhosis. J Autoimmun 41:87-91 (2013).

Loftfield E, Zhou W, Graubard BI, Yeager M, Chanock SJ, et al: Predictors of mosaic chromosome $\mathrm{Y}$ loss and associations with mortality in the UK Biobank. Sci Rep 8:12316 (2018).

Loftfield E, Zhou W, Yeager M, Chanock SJ, Freedman ND, Machiela MJ: Mosaic Y loss is moderately associated with solid tumor risk. Cancer Res 79:461-466 (2019).

Maan AA, Eales J, Akbarov A, Rowland J, Xu X, et al: The Y chromosome: a blueprint for men's health? Eur J Hum Genet 25:1181-1188 (2017).

Machiela MJ, Dagnall CL, Pathak A, Loud JT, Chanock SI, et al: Mosaic chromosome Y loss and testicular germ cell tumor risk. J Hum Genet 62:637-640 (2017)

Mangs AH, Morris BJ: The human pseudoautosomal region (PAR): origin, function and future. Curr Genomics 8:129-136 (2007).

Mannucci A, Sullivan KM, Ivanov PL, Gill P: Forensic application of a rapid and quantitative DNA sex test by amplification of the X-Y homologous gene amelogenin. Int J Legal Med 106:190-193 (1994).

Milsted A, Underwood AC, Dunmire J, DelPuerto HL, Martins AS, et al: Regulation of multiple renin-angiotensin system genes by Sry. J Hypertens 28:59-64 (2010). 
Morikawa T, Yamamoto Y, Miyaishi S: A new method for sex determination based on detection of SRY, STS and amelogenin gene regions with simultaneous amplification of their homologous sequences by a multiplex PCR. Acta Med Okayama 65:113-122 (2011).

Noveski P, Madjunkova S, Sukarova Stefanovska E, Matevska Geshkovska N, Kuzmanovska M, et al: Loss of $\mathrm{Y}$ chromosome in peripheral blood of colorectal and prostate cancer patients. PLoS One 11:e0146264 (2016).

Paracchini S, Pearce CL, Kolonel LN, Altshuler D, Henderson BE, Tyler-Smith C: A Y chromosomal influence on prostate cancer risk: the multi-ethnic cohort study. J Med Genet 40: 815-819 (2003).

Persani L, Bonomi M, Lleo A, Pasini S, Civardi F, et al: Increased loss of the $\mathrm{Y}$ chromosome in peripheral blood cells in male patients with autoimmune thyroiditis. J Autoimmun 38:J193-196 (2012).

Phillips C: Forensic genetic analysis of bio-geographical ancestry. Forensic Sci Int Genet 18: 49-65 (2015).

Plaseska Karanfilska D, Noveski P, MatevskaGeshkovska N, Dimovski A, Efremov GD: Y chromosome haplogroup R1a is associated with prostate cancer risk among Macedonian males. Conference Paper, European Human Genetics Conference, Vienna, Austria (2009).

Prinz M, Sansone M: Y chromosome-specific short tandem repeats in forensic casework. Croat Med J 42:288-291 (2001).

Prokop JW, Tsaih SW, Faber AB, Boehme S, Underwood AC, et al: The phenotypic impact of the male-specific region of chromosome-Y in inbred mating: the role of genetic variants and gene duplications in multiple inbred rat strains. Biol Sex Differ 7:10 (2016).

Roewer L: Y-chromosome short tandem repeats in forensics-sexing, profiling, and matching male DNA. WIREs Forensic Sci 1:e1336 (2019).

Sauter G, Moch H, Wagner U, Novotna H, Gasser TC, et al: Y chromosome loss detected by FISH in bladder cancer. Cancer Genet Cytogenet 82:163-169 (1995).
Schmittgen TD, Livak KJ: Analyzing real-time PCR data by the comparative $\mathrm{C}_{\mathrm{T}}$ method. Nat Protoc 3:1101-1108 (2008).

Shewale JG, Liu RH: Forensic DNA Analysis: Current Practices and Emerging Technologies (CRC Press, Boca Raton 2016).

Silva Veiga LC, Bérgamo NA, Reis PP, Kowalski LP, Rogatto SR: Loss of Y-chromosome does not correlate with age at onset of head and neck carcinoma: a case-control study. Braz J Med Biol Res 45:172-178 (2012).

Sinclair AH, Berta P, Palmer MS, Hawkins JR, Griffiths BL, et al: A gene from the human sex-determining region encodes a protein with homology to a conserved DNA-binding motif. Nature 346:240-244 (1990).

Singh NP, Madabhushi SR, Srivastava S, Senthilkumar R, Neeraja C, et al: Epigenetic profile of the euchromatic region of human Y chromosome. Nucleic Acids Res 39:3594-3606 (2011).

Skaletsky H, Kuroda-Kawaguchi T, Minx PJ, Cordum HS, Hillier L, et al: The male-specific region of the human $\mathrm{Y}$ chromosome is a mosaic of discrete sequence classes. Nature 423:825837 (2003).

Sobrino B, Brión M, Carracedo A: SNPs in forensic genetics: a review on SNP typing methodologies. Forensic Sci Int 154:181-194 (2005).

Strimbu K, Tavel JA: What are biomarkers? Curr Opin HIV AIDS 5:463-466 (2010).

Terao C, Momozawa Y, Ishigaki K, Kawakami E, Akiyama M, et al: GWAS of mosaic loss of chromosome Y highlights genetic effects on blood cell differentiation. Nat Commun 10: 4719 (2019).

Thompson DJ, Genovese G, Halvardson J, Ulirsch JC, Wright DJ, et al: Genetic predisposition to mosaic Y chromosome loss in blood. Nature 575:652-657 (2019)

Tilford CA, Kuroda-Kawaguchi T, Skaletsky H, Rozen S, Brown LG, et al: A physical map of the human Y chromosome. Nature 409:943945 (2001).
Tozzo P, Giuliodori A, Corato S, Ponzano E, Rodriguez D, Caenazzo L: Deletion of amelogenin Y-locus in forensics: literature revision and description of a novel method for sex confirmation. J Forensic Leg Med 20:387-391 (2013).

Veerappa AM, Padakannaya P, Ramachandra NB: Copy number variation-based polymorphism in a new pseudoautosomal region 3 (PAR3) of a human X-chromosome-transposed region (XTR) in the Y chromosome. Funct Integr Genomic 13:285-293 (2013).

Vijayakumar S, Garcia D, Hensel CH, Banerjee $\mathrm{M}$, Bracht $\mathrm{T}$, et al: The human $\mathrm{Y}$ chromosome suppresses the tumorigenicity of PC-3, a human prostate cancer cell line, in athymic nude mice. Genes Chromosomes Cancer 44:365372 (2005)

Wang YC, Ma XY, Sun XF, Xian JJ, Li SY, et al: Alleles dropout patterns of Y-short tandem repeats in infertile males with $\mathrm{Y}$ chromosome microdeletions (in Chinese). Yi Chuan 41: 243-253 (2019).

Wong JYY, Margolis HG, Machiela M, Zhou W, Odden MC, et al: Outdoor air pollution and mosaic loss of chromosome $\mathrm{Y}$ in older men from the Cardiovascular Health Study. Environ Int 116:239-247 (2018).

Wright DJ, Day FR, Kerrison ND, Zink F, Cardona A, et al: Genetic variants associated with mosaic Y chromosome loss highlight cell cycle genes and overlap with cancer susceptibility. Nat Genet 49:674-679 (2017).

Yuan X, Lu ML, Li T, Balk SP: SRY interacts with and negatively regulates androgen receptor transcriptional activity. J Biol Chem 276: 46647-46654 (2001)

Zhou W, Machiela MJ, Freedman ND, Rothman $\mathrm{N}$, Malats N, et al: Mosaic loss of chromosome $\mathrm{Y}$ is associated with common variation near TCL1A. Nat Genet 48:563-568 (2016).

Zuo E, Huo X, Yao X, Hu X, Sun Y, et al: CRISPR/ Cas9-mediated targeted chromosome elimination. Genome Biol 18:224 (2017). 